\title{
Gravitational and Quantum Effects in Neuron Function
}

\author{
Gustavo Rodrigues Ferreira Gomes \\ International Center of Condensed Matter Physics, University of Brasilia (UnB), Brasilia, 70297-400, Distrito Federal, Brazil
}

\begin{abstract}
The relation between microtubules architecture in the cytoskeletal structure inside the dendrites and soma and the emergence of neuron function and firing action potential crosses the tiny line between physics and biology. As decoherence is a fundamental mechanism in some biological process such as photosynthesis and others examples, the gravitational quantum approach may contribute to elucidate if neuron function really emerges from quantum coherence in neuronal microtubules. The Einstein equation correlates the stress-energy tensor $T_{\mu \nu}$ to a specific divergence-free combination Ricci tensor $R_{\mu \nu}$ and the metric. In the semiclassical formulation, we have $\mathrm{G}_{\mu \nu} \equiv R_{\mu \nu}-\frac{1}{2} g_{\mu \nu} R=\frac{8 \pi G}{c^{4}}\left\langle\psi\left|\widehat{T}_{\mu \nu}\right| \psi\right\rangle$ which describes the quantum field in curved space-time geometry. But for a more precise equation in relation to the stress-energy tensor, we know that in a non-zero temperature, the wave-function is not enough to describe the physical reality. A more precise equation demands a formulation in the density-matrix form, $\rho$, but for now there is no Diósi-Penrose model with density-matrix formulation. Such a density-matrix description can be viewed as a probability mixture of different wave-functions. Using some algebra and rules related to the mathematical manipulation of the density-matrix applied to operators, such the stress energy tensor, we found the von Neumann-Einstein equation for the general relativity equation in the density matrix operator form, $\mathrm{G}_{\mu \nu}=\frac{8 \pi G}{c^{4}} \operatorname{Tr}\left[\rho \widehat{T}_{\mu \nu}\right]$. Thus density-matrix operator-instead of just a wave function of pure states - applied to the stress-energy tensor gives the curvature of space time, given by Einstein tensor, $\mathrm{G}_{\mu \nu}$. The quantum fluctuation in the gravitational space-time field might feed back to decohere the quantum density-matrix. As long as decoherence can be viewed as the loss of information from a system to the environment, the density-matrix $\rho$ is also related to that process and considering the measurement problem, density-matrix $\rho_{\text {after }}$ is a more complete description of the possible outcome of the measurement. It is possible that some characteristics of the special microtubulin-associated proteins (MAP) that capes the dendritic-somatic microtubulins which could induces longer-lived nuclear spin states prevented from de-polymerization and suitable for long term information encode and memory. Understand the mechanism by which the hyper-phosphorylation in type tau-MAP displacements from microtubulins results in neurofibrillary tangles and cognitive dysfunctions in Alzheimer's disease.
\end{abstract}

Key words: Semiclassical equation, quantum gravity, decoherence, microtubulin, neuron function.

\section{Background}

The mathematic and physical fundaments of the brain function have become more evident along with the deeper understanding of physical universe and the new top discoveries in astrophysicist and cosmology appear to play an important and fundamental role in the establishment of physical structure of human consciousness. Beginning with most abstract science as mathematics, the Gödel's first incompleteness theorem states that no consistent system of axioms is capable of algorithmic proving all the truth about the system, in other words, there are always statements that are true,

Corresponding author: Gustavo Rodrigues Ferreira Gomes, research field: quantum gravitation. but cannot be proven within the axioms of the system. Furthermore, no system can demonstrate its own consistency [1]. Penrose, in 2014 argued that some conscious features such as understanding are not algorithmic computable and this incompleteness relates to the incomplete laws of physics at the scale of the brain which leads to an incompleteness of quantum theories while involving the "measurement problem" [2]. The measurement problem is related to the unitary evolution which is a continuous deterministic evolution of quantum state and the measurement-observation-of the system collapses the quantum states. This problem could be seen as the famous Schrödinger's cat. One interpretation is that the objective reduction (collapse of the wave function) 
occurs when some objective threshold is achieved [3]. Maybe the objective reduction occurs when a tiny amount of information (or qubit) is transferred from the system to another system, decreasing the probability density function $\Psi$ from a set of all eigenvectors in the Hilbert infinite space to one specific classical eigenstate, caused by the principle of conservation of information.

Diósi and Penrose have proposed an extension (known as DP) of current quantum mechanics, taking the bridge between quantum- and classical-level as a 'quantum-gravitational' phenomenon. They have stated that the objective reduction of the wave function to a certain eigenstate is due to Einstein's general relativity in which the superposed time-evolution of the gravitational location of the particle are caused by quantum fluctuations in the space-time location. They calculate the time $\tau$ to the collapse of the superposition according to the formula $\tau \approx \hbar / E_{G}$ where $\hbar$ is the Dirac's form of Planck's constant $\mathrm{h}$ and $E_{G}$ is the gravitational self-energy of the difference between the two stationary mass distributions of the superposition (Fig. 1). This time $\tau$ appears to be related to the appearance of a 'decoherence' in which objective reduction of the probability density wave function $\Psi$ are caused by gravitational action in the "quantum"-space-time.

According to Hodgkin and Huxley model, neurons are "integrate-and-fire" threshold logic devices in which branched dendrites and soma receive, integrate and resend synaptic inputs. Interiors of eukaryotic cells are organized by their cytoskeleton, microtubule-associated proteins (MAPs), actin and intermediate filaments. Microtubules (MTs) are cylindrical polymers. The hyper-phosphorylated tau MAP is said to be related to the displacement from MTs results in neurofibrillary tangles and the cognitive impairment of Alzheimer's disease. This mechanism could be accessed by electron crystallography.

In microtubulins, coherent states emerges with electric dipole due to coupled London force attraction among indole and phenyl rings of aromatic amino acids such as tyrosine, phenylalanine and tryptophan. The oscillation between alternate states becomes a superposition of both states as a qubit. These quantum channels occur within microtubules subunit proteins and between the tubulins along helical pathways through microtubule lattice (Fig. 2). Then, it is speculated that the gravitational force coupled with the uncertainty of the van der Walls London dipole can drive the collapse of Schrödinger's wave function which occurs in time $\tau$. Hameroff stated that time $\tau$ is of order of miliseconds, but there are evidences that it could be even fast (10 picoseconds) because all protein catalytic function should have a survival value in order the complexity within the cell to be organized against the increase of entropy with time. Since the tubulins cannot control the voltage-gated ion-channels it should

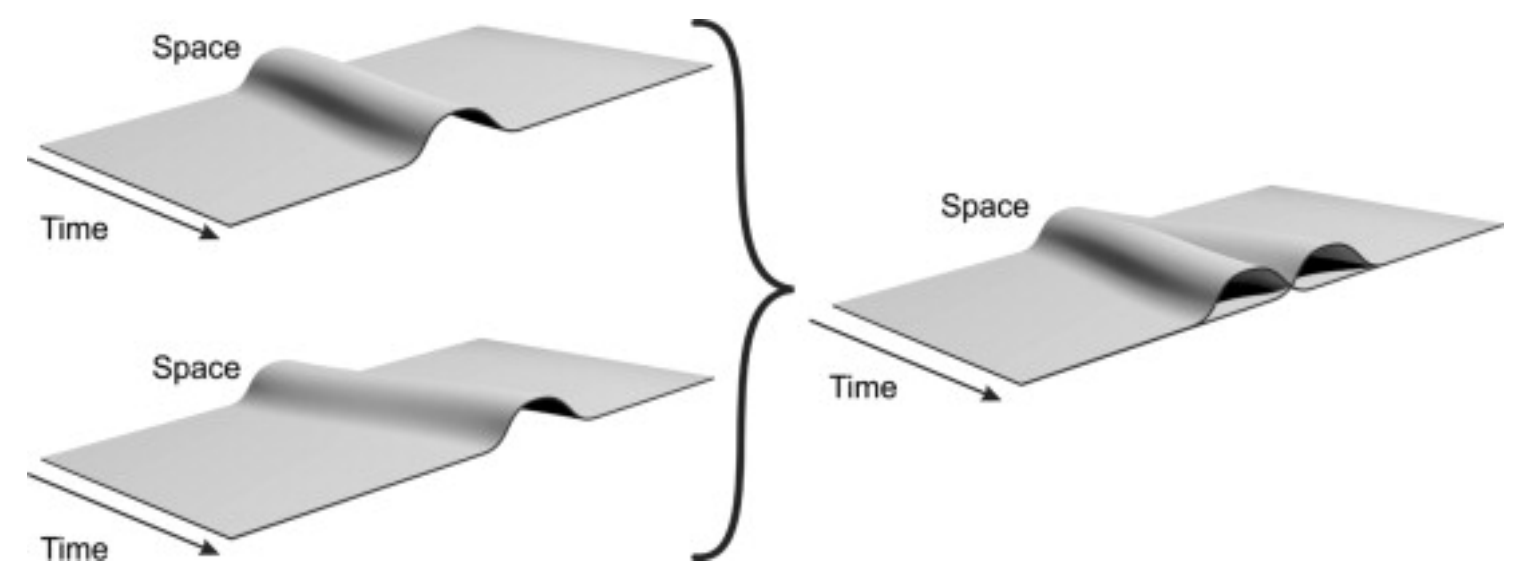

Fig. 1 Left: independent evolution of two alternative particle locations. Right: Quantum superposition of both particle locations. 

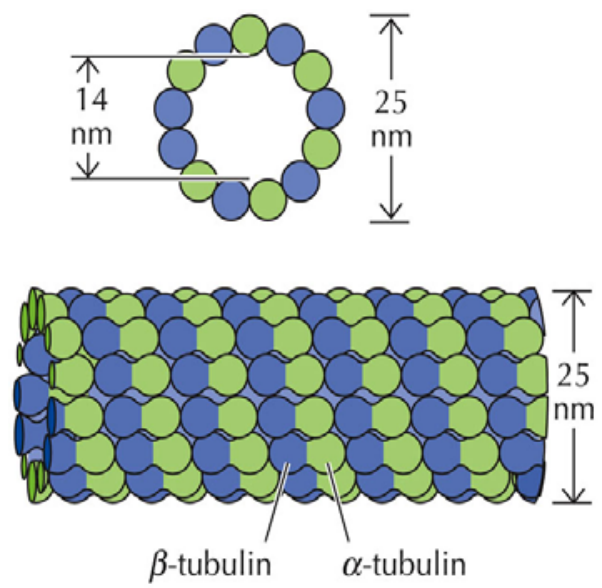

Fig. 2 Structure of a Microtubule and its tubulins.

control it by affecting the release of neurotransmitters from vesicle to the synaptic cleft. MT information processing appear to be related to the context of cellular ("molecular") automata ("microtubule automata") in which tubulin dipole London force attractions and conformational states interact with neighbor tubulin states in hexagonal MT lattices by dipole couplings, synchronized by biomolecular coherence as proposed by Fröhlich [4]. In photosynthetic organisms the quantum coherence is involved in transfer energy in time scale predicted by quantum biology [5]. Sarovar and Akihito, have identified entanglement and quantum coherence between excited states of pigments in photosynthesis [6]. Bio-conductor material acts in quantum transport which plays an important role in evolution and selection, because it represents a new distinct way of transport in quantum biological systems [7].

Tubulin is composed of a heterogeneous group of amino acids residues connected to peptide backbones and this residues include both water insoluble non-polar groups and water-soluble polar, the first including "aromatic" amino acids (phenylalanine, tyrosine and tryptophan) with " $\pi$ " orbital electron resonance clouds in phenyl and indole rings. $\pi$ orbital clouds are composed of electrons able to delocalize across a spatial region [8]. Like oil separating from water, non-polar electron clouds coalesce during protein folding to form isolated water-excluding "hydrophobic regions" within proteins with particular ("oily", "lipid-like") solubility. Driving the folding are non-polar, but highly polarizable $\pi$ orbital electron cloud dipoles which couple by van der Waals London forces (instantaneous dipole-induced dipole attractions between electron clouds) [9].

Topological quantum computation have shown A-lattice hydrophobic quantum channels in helical winding pattern matching Fibonacci geometry (Fibonacci series in which each number is the sum of the two preceding numbers, e. g. 1, 1, 2, 3, 5, 8 ..., Fig. 3). Bandyopadhyay has found evidence for ballistic conductance and quantum interference along helical pathway lattice [10]. Other mechanisms could include chain correlated spin along lattice pathway and the periodic spin-flip or spin precession processes in the context of alternate current at specific frequencies. The produced patterns could also determine MAP attachment sites and thus govern intra-neuronal architecture and synaptic function by modulating sensitivity of membrane receptors, ion channels, exoplasmic transport and communication with genetic material.

\section{Gravitational Effects}

Space-time coordinates and matter degrees of freedom in the matrix formulation in a noncommutative generalization of special relativity is described by the line-element [11]

$$
d s^{2}=\operatorname{Tr} d \hat{s}^{2} \equiv \operatorname{Tr}\left[d \hat{t}^{2}-d \hat{x}^{2}-d \hat{y}^{2}-d \hat{z}^{2}\right]
$$

Then we have the semiclassical Einstein equation

$$
\mathrm{G}_{\mu \nu} \equiv R_{\mu \nu}-\frac{1}{2} g_{\mu \nu} R=\frac{8 \pi G}{c^{4}}\left\langle\psi\left|\widehat{T}_{\mu \nu}\right| \psi\right\rangle
$$

that describes the quantum field in curved space-time geometry. In the first place, it should be stressed that in standard quantum treatments of systems at non-zero temperature, the description would be in terms of a density-matrix rather than a simple wave-function. The wave-function is appropriate just for pure states. Such a density-matrix description can be viewed as a 

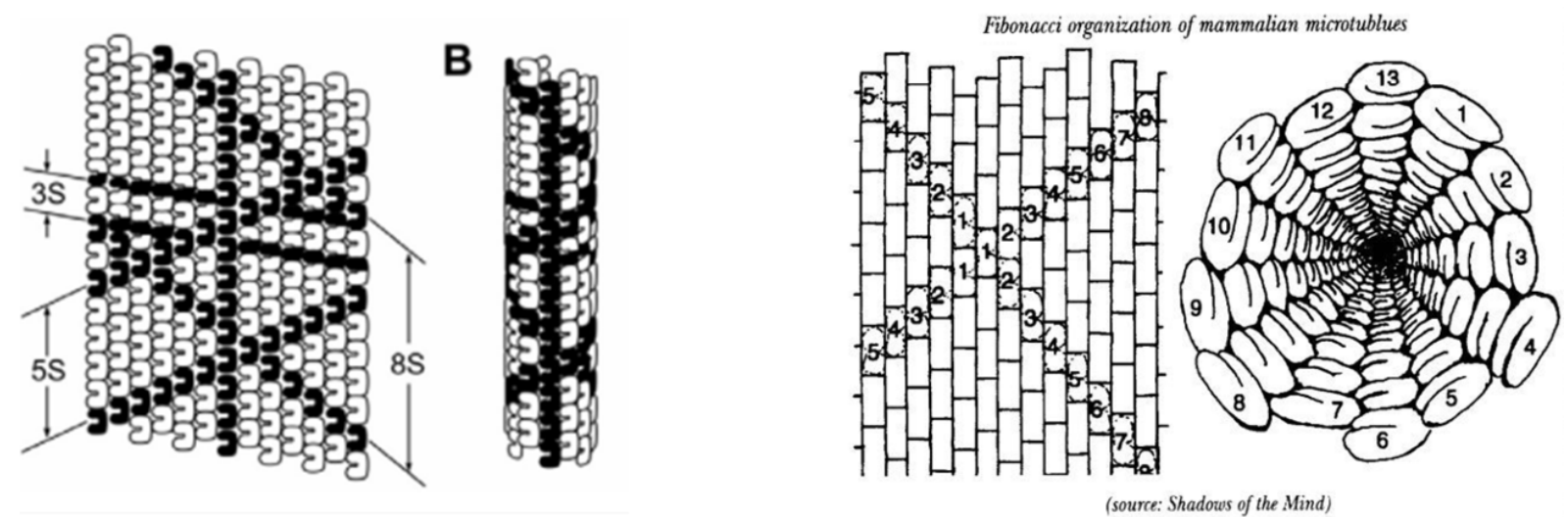

Fig. 3 Extending microtubule results in Fibonacci geometry of hydrophobic channels. Dipole coupled London forces in hydrophobic channels superimpose a slight separation of atomic nuclei $E_{G}$ sufficient to objective reduction.

probability mixture of different wave-functionsalthough a single density-matrix can be interpreted in many different ways as such a probability mixture. As yet, a fully appropriate generalization of the Diósi-Penrose scheme to a density-matrix description has not been provided [12].

For a first attempt, we use the procedure for extracting a meaningful, finite part from the formally divergent expectation value of the stress tensor. One possible choice is to separate the space-time points at which the fields in $\widehat{T}_{\mu \nu}$ are evaluated, and then to average over the direction of separation. This leaves $\left\langle T_{\mu \nu}\right\rangle$ depending upon an invariant measure of the distance between the two points. These same solutions fragmented slightly, releasing pulses carrying a small fraction of their total probability to infinity, while the rest of their amplitude remained more localized [13].

\section{Development}

Then for a density-matrix in von Neumann formulation, we have

$$
\rho=\sum_{i} p_{i}\left|\psi_{i}\right\rangle\left\langle\psi_{i}\right|
$$

and

$$
|\psi\rangle=\sum_{i} p_{i}\left|\psi_{i}\right\rangle \text { where } T\left|\psi_{i}\right\rangle=\widehat{T}\left|\psi_{i}\right\rangle
$$

and $\sum_{i}\left|p_{i}\right|^{2}=1$. For an observable tensor stress-energy $T_{\mu \nu}$ the expected value $\left\langle T_{\mu \nu}\right\rangle$ is

$$
\begin{gathered}
\left\langle T_{\mu \nu}\right\rangle=\left\langle\psi\left|\widehat{T}_{\mu \nu}\right| \psi\right\rangle \\
=\sum_{i} p_{i}\left\langle\psi_{i}\left|\widehat{T}_{\mu \nu}\right| \psi_{i}\right\rangle \\
=\sum_{i} p_{i}\left\langle\psi_{i}\left|\widehat{T}_{\mu \nu} \sum_{n}\right| \psi_{n}\right\rangle\left\langle\psi_{n}|| \psi_{i}\right\rangle \\
=\sum_{n} \sum_{i} p_{i}\left\langle\psi_{n} \mid \psi_{i}\right\rangle \widehat{T}_{\mu \nu}\left\langle\psi_{i} \mid \psi_{n}\right\rangle \\
=\operatorname{Tr}\left[\sum_{i} p_{i}\left|\psi_{i}\right\rangle\left\langle\psi_{i}\right| \widehat{T}_{\mu \nu}\right] \\
\left\langle T_{\mu \nu}\right\rangle=\operatorname{Tr}\left[\rho \widehat{T}_{\mu \nu}\right]
\end{gathered}
$$

Plugin this equation in Einstein quantum field equation we get the von Neumann-Einstein equation for the general relativity equation in the density-matrix operator form

$$
\mathrm{G}_{\mu \nu}=\frac{8 \pi G}{c^{4}} \operatorname{Tr}\left[\rho \widehat{T}_{\mu \nu}\right]
$$

Thus density-matrix operator - instead of just a wave function of pure states - applied to the stress-energy tensor gives the curvature of space time, given by Einstein tensor, $\mathrm{G}_{\mu \nu}$. For a quantum stress-energy tensor of a superposed quantum state the density-matrix causes the entanglement in which the off-diagonal elements vanish. Also, the $\operatorname{Tr}\left[\rho \widehat{T}_{00}\right]$ corresponds to density mass and $\operatorname{Tr}\left[\rho \hat{T}_{i i}\right]$, where $i=1,2,3$ corresponds to pressure. The quantum fluctuation in the gravitational space-time field might feed back to decohere the quantum density-matrix [14]. 


\section{Quantum Effects}

Decoherence can be viewed as the loss of information from a system to the environment. So the decay in quantum information caused by the entanglement of the system to the environment is also called einselection [14]. The quantum decoherence actually decreases information by erasing interference in the composite system. The quantum decoherence occurs when different elements of a system are separated from each other in a process called einselection (environment induced superselection) possibly occurs when a certain gravitational threshold is reached in an objective reduction event (OR). The uncertain related to density matrix applied to the stress-energy tensor appears as uncertain lumps in the space-time curvature $\mathrm{G}_{\mu \nu}$.

For an observable $\mathrm{A}$ we apply the spectral decomposition $A=\sum_{n} \alpha_{n} P_{n}$, where $P_{n}$ satisfies the closure or completeness relation $\sum_{n} P_{n}=1$. The measurement of this observable $\mathrm{A}$ is described by the eigenprojectors of A. A system prepared in state $\rho$ has the possible outcomes of the measurement

$$
\rho_{\text {after }}=\frac{P_{n} \rho P_{n}}{\operatorname{Tr}\left[P_{n} \rho P_{n}\right]}
$$

And this correlates decoherence and einselection process. Relaxation and noise are caused by the environment perturbing the system, while decoherence and einselection are caused by the system perturbing the environment [15].

\section{Discussion}

As discussed above, the human consciousness may be related in some way to the quantum decoherence process occurring in neuronal microtubules, especially in giant pyramidal layer $\mathrm{V}$ of the pre-frontal neurons. The other important question is if gravity, through general relativity, may interfere in quantum process, such as decoherence. Equation 1 is a tiny indicative that this could be the case. DP model could be expanded to englobe the general relativity approach and density-matrix. This is a more "down-to-earth" viewpoint of environmental decoherence, in which interaction of a superposition with its environment "erodes" quantum states, so that instead of a single wave function being used to describe the state, a more complex entity is used.

Back to biology, according to the tau hypothesis, in Alzheimer's disease, the excessive and abnormal phosphorylation of tau-MAP results into tau paired helical filament and neurofibrillary tangles which disrupt microtubules and disintegrate the structure of the cell's cytoskeleton leading to the initial loss of memory and later to the death of the neuron and loss of cognitive functions. Zhang et al. in 2015 have performed a detailed analysis on changes in posttranslational modifications of alpha-tubulins and shown a compensatory modification in Alzheimer's disease. Other causes have been proposed such as guanosine triphosphate binding to beta-subunit of tubulin [16]. The tau-MAP hyper-phosphorylation has shown to be one the most prominent line of investigation in Alzheimer's disease. Electron crystallography could be used to elucidate the mechanisms by which the clustered arrays of electron resonance rings becomes the "quantum channels" and if it could induce the longer-lived nuclear spin states for short-term memory.

\section{Conclusions}

The coherent state of neurotubulins heterodimers is the site of action of the anesthetics gas molecules in prevention of consciousness and memory and consequently may play an important role in degenerate diseases, such as Alzheimer. Possibility of interaction of the coherent microtubulin heterodimers with formation, modulation e regulation of the neural action potential must be investigated. It has been proposed that the coherent states of these microtubulin heterodimers produce patterns that could also modulate sensitivity of ion channels, exoplasmic transport and membrane receptors, thus governing intra-neuronal architecture and synaptic formation and function. 
Additionally, neurotubulins is involved in most of degenerative disease such as Parkinson, Alzheimer and other diseases [17] and the deeper comprehension of the relation between tubulins defects and impairment of cognitive function could facilitate the development of medicines and alternative treatments to this diseases.

As pointed out by Bandyopadhyay: "microtubule bundles of two neurons vibrate resonantly and exchange energy; the mismatchcauses the firing" [18]. This resonance may be related to decoherence time $\tau$ in these microtubules, while transferring quantum information. The deepening in the study of the relation between gravitational quantum effects and decoherence may cast light in the understanding of the possibility of these process also happen in the neuron and the brain. As long as quantum effects are fundamental mechanism in some biological process such as photosynthesis and other examples, and based on experimental evidences the gravitational quantum approach may contribute to elucidate if neuron function is really related to quantum decoherence in neuronal microtubules.

\section{Reference}

[1] Lindström, P. 1997. Aspects of Incompleteness Lecture Notes Logic, vol. 10. Springer.

[2] Hameroff, S., and Penrose R. 2014. "Consciousness in the universe: a review of the 'Orch OR' theory." Phys. Life Rev. 11 (1): 39-78.

[3] Percival, I. C. 1994. "Primary state diffusion." Math Phys Sci, Quantum Communications and Measurements 447: 189-209.

[4] Fröhlich, H. 1968. "Long range coherence and energy storage in biological systems." Int. J. Quant. Chem. 2: 641-9.
[5] Engel, G. 2007. "Evidence for wavelike energy transfer through quantum coherence in photosynthetic systems." Nature 446 (7137): 782-6.

[6] Sarovar, M., and Ishizaki, A. 2010. "Quantum entanglement in photosynthetic light-harvesting complexes." Nature Physics 6 (6): 462-7.

[7] Vattay, G., and Salahub, D. 2015. "Quantum Criticality at the Origin of Life." Journal of Physics, Conference Series 626012023.

[8] Voet, D., and Voet, J. 1995. Biochemistry (2nd edition), Wiley.

[9] Hameroff, S. 1998. "Anesthesia, consciousness and hydrophobic pockets - a unitary quantum hypothesis of anesthetic action." Toxicol Letters 100-101: 31-9.

[10] Bandopadhyay, A. 2011. "Direct experimental evidence for quantum states in microtubules and topological invariance." In Towards a Science of Consciousness, Stockholm, Sweden.

[11] Tejinder, P. Singh, 2015. "Possible role of gravity in collapse of the wave-function: a brief survey of some ideas." Journal of Physics, Conference Series 626 012009.

[12] Pfaff, W. et al., 29 May 2014. "Unconditional quantum teleportation between distant solid-state quantum bits." Science.

[13] van Meter, J. R. 2011."Schrödinger-Newton collapse of the wave function". Class. Quant. Gravit. 28, 215013.

[14] Zurek, W. H. 2003. "Decoherence, einselection, and the quantum origins of the classical." Reviews of Modern Physics 75: 715-65.

[15] Zurek, W. H. 2002. "Decoherence and the transition from quantum to classical-revisited.” Los Alamos Science 27: 86-109.

[16] Khatoon, S., and Grundke-Iqbal, I. 1995. "Guanosine triphosphate binding to beta-subunit of tubulin in Alzheimer's disease brain: role of microtubule-associated protein tau." J. Neurochem 64 (2): 777-87.

[17] Lei, P., Ayton, S., Finkelstein, D. I., November 2010. "Tau protein: relevance to Parkinson's disease." Int. J. Biochem Cell Biol. 42 (11): 1775-8.

[18] Bandopadhyay, A. 2016, Personal Communication. 